\title{
The Effects of Cynomorium songaricum on the Reproductive Activity in Male Golden Hamsters
}

\author{
Jee Soo Lee, Hyun Ah Oh, Ji Young Kwon, Min Ho Jeong, Jong Seok Lee, \\ Dong Won Kang and Donchan $\mathrm{Choi}^{\dagger}$ \\ Department of Life Science, College of Environmental Sciences, Yong-In University, Yongin 449-714, Korea
}

\begin{abstract}
Cynomorium songaricum (CS) has been used in traditional Korean medicine in treating male impotence and sexual dysfunction. We investigated the effects of aqueous CS extract on the reproductive activity of golden hamsters whose spermatogenetic capacity is active in summer and inactive in winter. The animals were divided into 5 groups: long photoperiod (LP) control, short photoperiod (SP) control, and SP animals treated with low, middle, or high concentrations of CS. The animals were orally ingested with low $(0.5 \mathrm{~g} / \mathrm{kg})$, middle $(1.0 \mathrm{~g} / \mathrm{kg})$, or high $(2.5 \mathrm{~g} / \mathrm{kg})$ concentrations of the aqueous extracts for 8 weeks on the daily basis. The control animals received the vehicle. As results, the LP control animals showed active testicular function but SP control animals displayed remarkably reduced testicular weights. The outcomes of the reproductive activity from low and middle concentrations of CS treatments were identical and marked as low dose. The consequences were a partial blocking of regressing activity by SP. On the other hand, the animals treated with high dose of CS extract showed remarkable significance in comparison to the SP control, indicative of a complete blocking effect of the CS on the regressing testes by SP. There were a dose-dependent effects of the CS on the sexual function. These results suggest that the CS extract promotes the male fertility by strengthening the spermatogenesis in the golden hamsters.

Key words : Cynomorium songaricum, Reproductive activity, Photoperiod, Golden hamster
\end{abstract}

\section{INTRODUCTION}

Cynomorium songaricum Ruprecht (CS), a parasitic perennial flowering plant, grows in dry, rocky, or sandy soil at high altitude, largely in China, Inner Mongolia, and Tibet. The CS that dried the rhizome and processed in a variety ways has been known to cure the poor sexual enhancement and to strengthen aphrodisiac function and immune activity (Nickrent et al., 2005). Moreover, it was reported that the ethanolic extract of the CS activated the female sex steroid production (Zhang et al., 2005). Also, in the light of the results that the CS increased the number of spermatozoa in the epididymis and testicular weight, the CS can be interpreted to promote the process of spermatogenesis and the potency of male fertility (Abdel-Rahman et al.,

\footnotetext{
${ }^{+}$Corresponding author: Donchan Choi, Dept. of Life Science, College of Environmental Sciences, Yong-In University, Yongin 449-714, Korea. Tel: +82-31-8020-2781, Fax: +82-31-8020-2886, E-mail: dcchoi@yongin.ac.kr
}

1999; Abdel-Magied et al., 2001; Yang et al., 2010). However, there has been no report about research whether the CS has a potential to prevent from the testicular regression of males. This study was focused on the effects of the CS extract in the course of the spermatogenesis of male golden hamsters.

Reproductive capacity is presented with unique characteristics from species to species. Almost all the animals who largely inhabit in temperate zone of the Earth do not possess the reproductive function throughout a year (Choi, 1996). This is because a vast amount of energy is required to maintain the active sexual activity. Thus most of animals reproduce for a particular period of time in a year, spare the energy, and keep the species (Choi \& Han, 2010; Choi \& Lee, 2012).

The golden hamster is a small rodent species whose reproductive inability is temporarily emerged for winter season in environment. The golden hamster is one of the 
seasonal breeders whose reproductive activities are active around summer season (Choi \& Lee, 2012). In the winter climate the reproductive functions are completely arrested. This seasonal breeding strategy is determined by photoperiod that is the length of lighting in a day, which is surmised that the animal can predict possibly the annual cycle in a period of a year.

The fluctuating changes of seasonal reproductive function in the hamsters can be reproduced in the artificial lighting regime. When the reproductively mature male hamsters are transferred to short photoperiod (SP, equals to and less than 12 hours of lights in a day), they lose generative activities, showing no functional spermatozoa in testes. If the length of lighting in a day is set to more than 12.5 hours (long photoperiod; LP) mimicking summer season, the generative functions are energetically resumed, doubtlessly promoted, and maintained afterward.

The goal of the present work was to investigate the effects of the CS extracts on the spermatogenesis in the course of testicular regression of male golden hamsters.

\section{MATERIALS AND METHODS}

\section{Plant material and extraction}

Cynomorium songaricum was procured from the local company in Korea (Hansol Pharmacy Co., Ltd.). The extraction was carried out by mixing the fragmented pieces of the CS with 1:20 w/v in drinking water for two weeks. The mixture was squeezed with woven texture to collect the fluid. The fluid was evaporated to decoct by warming up the vessel containing the fluid in hot water. Then it was dried in the oven kept at $60^{\circ} \mathrm{C}$. The solidified product was milled to fine powder by Osterizer blender. The yield of the extract was $26.0 \% \mathrm{w} / \mathrm{w}$ in terms of dried starting material weights. The powered extract was light brownish. The extract was preserved in a refrigerator, and was dissolved with drinking water prior to usage.

\section{Animals}

Adult male golden hamsters (Mesocricatus auratus) weighing 80-100 g were used for the study. They were housed in plastic cages under LP conditions of light and dark (light of 14 hours : darkness of 10 hours) with an ambient temperature of $22 \pm 1^{\circ} \mathrm{C}$. Reproductive activity of these hamsters are always active in the photoperiod. They were fed with standard laboratory mouse chow and tap water $a d$ libitum. The condition of management of animals was approved by the Yongin University Institutional Animal Care and Use Committee (YUIACUC-2012-04).

\section{Photoperiod treatment and intubation of the CS}

The golden hamsters were divided into five groups. The control animals were maintained in LP (light of 14 hours : darkness of 10 hours) or SP (light of 10 hours : darkness of 14 hours) and received the vehicle (drinking water). The experimental animals were housed in SP. The CS extract was dissolved in vehicle and was administered orally each day for 56 consecutive days using an intubation needle. The animals received the CS extract at doses of $0.5 \mathrm{~g}, 1.0$ $\mathrm{g}$, or $2.5 \mathrm{~g}$ per $\mathrm{kg}$ of body weights on a daily basis. During this experimental period the body weights were measured every week. The testicular weights were determined at 0 , 4 , and 8 weeks. At the end of treatment, the animals were decapitated. Testicular volumes were measured, were isolated, and were actually weighed to compare the relationship between the weights calculated from the measured values and real weights by actual weighing. The weights of testes were converted from the testicular volumes that was calculated from the measurements of the major axis and the minor axis (Watson-Whitmyre \& Stetson, 1985). The internal organs were also dissected and weighed. During the period of time, the behaviour of animals was observed to examine any abnormal phenomenon because of long-term experiment.

\section{Determination of testes weights}

The volume of testes was measured by laparotomy. The hamsters were anesthetized using diethyl ether with the aid of pentobarbital (25 mg/kg of body weight) and ketamine 
(50 $\mathrm{mg} / \mathrm{kg}$ of body weight). The laparotomy was rapidly performed when they were anesthetized. After excising the skin overlying the scrotal sac and protruding the testes within the scrotal sac, the major axis and the minor axis of testes were measured by vernier calipers. The skin excised was then sutured with surgical thread. The weights of the testes were calculated by the measurements. This method is greatly economic by reducing the animals without killing the many animals.

\section{Statistical analysis}

Data were expressed as mean \pm SEM. Statistical analysis was performed using Student's $t$-test. Differences were considered to be significant at $\mathrm{P}<0.05$.

\section{RESULTS}

The relationship between measured and weighed values of testes were identified by comparing the values calculated from the measures with the real values weighed. The correlation was shown in Fig. 1. The real values weighed on $\mathrm{X}$-axis and the converted values on $\mathrm{Y}$-axis demonstrated a positive relationship, and the correlation coefficient was 0.95 , close to 1 . The outcome appeared to be perfect correlation, meaning that the measured values were completely reflected by the weighed values. Although the density of same organs may not be identical as organs consisting of living body, it can be treated with minor differences in testicular weights whether they are measured or weighed. It is reasonable that low weights of testes, such as small testicular volume, display the reduced size of cells and decreased number of the cells within the organ. In the Fig. 1, the intercept point on $\mathrm{Y}$ axis was 0.30 that is near to the measured values. This means that the size of testes is likely to be gauged slightly longer than real mass of testes in evaluating the lengths (major and minor lengths) by exposing the testes within the scrotal sac in case of very small testes. It is apparent that the regressed testes with very small weights by involution resulted from some disorders

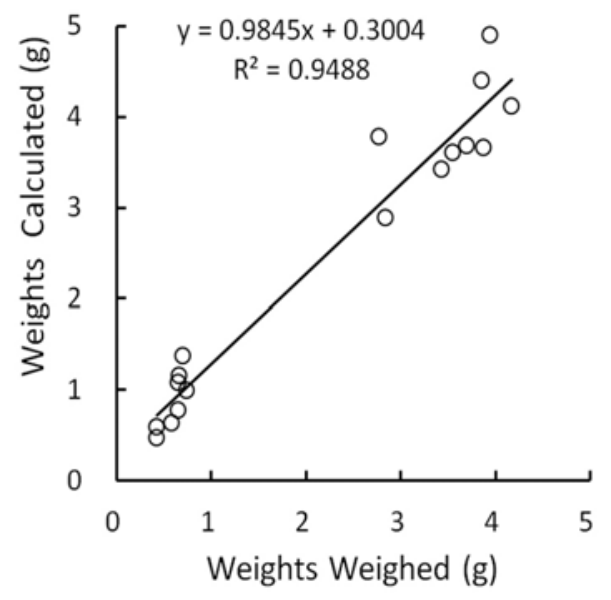

Fig. 1. A correlation between the testicular volume and the real testicular weights. Abscissa shows the real values weighed and ordinate the values calculated to the weights from the major and the minor measures. As the correlation coefficient is near to 1 , it could be estimated that the values converted from the volume exhibited the values weighed actually $(n=17)$.

in cell division.

There were no particular behavior in animals treated with the CS extract for 8 weeks. The change of body weights during the entire experimental period of time was matched the growth pattern. In general the body weights gradually increased from the beginning of the experiment. No significant differences were observed between groups at particular time point. Thus the CS extract was concluded not to affect body weights and any abnormal behavior.

The changes of testicular weights calculated from the major and minor axis measured were shown in Fig. 2 throughout the entire period of experiment. At the beginning the weights of testes were about $2.61 \pm 0.285 \mathrm{~g}$ (mean $\pm \mathrm{SEM}$ ). The testicular weights were steadily elevated in LP control but those in SP control had completely involuted testes in 8 weeks with slightly reduced at 4 weeks. The low and middle concentration of CS treatments provoked similarly partial blocking effects of regressing activity of SP, and they were combined (named SP CSL). The animals treated with high dose of the CS extract (named SP CSH) showed remarkable significance in com- 
parison to the SP control, indicative of a complete blocking effect of the CS on the regressing testes of SP. In 8 weeks of experiment, the testicular weights resulted in $3.79 \pm 0.036$ $\mathrm{g}$ in LP control, $0.37 \pm 0.265 \mathrm{~g}$ in SP control, $0.96 \pm 0.829 \mathrm{~g}$ in SP CSL, and $3.84 \pm 0.209 \mathrm{~g}$ in SP CSH. The mass of testes in SP control was reduced to virtually one tenth compared to that in LP control. One of the animals in SP CSL had fairly large mass of testes. The testicular weights of animals in SP CSL were much diminished but still significantly different from those of animals in SP control in the statistical aspects. As peculiar results, the testes in animals administered with high dose of the CS extract were not reduced at all, meaning a complete blockage of the influence of SP in golden hamsters.

At the end of experiment ( 8 weeks) the real weights of testes were in fact weighed (Fig. 3). The testicular weights were maintained large in LP control but those in SP

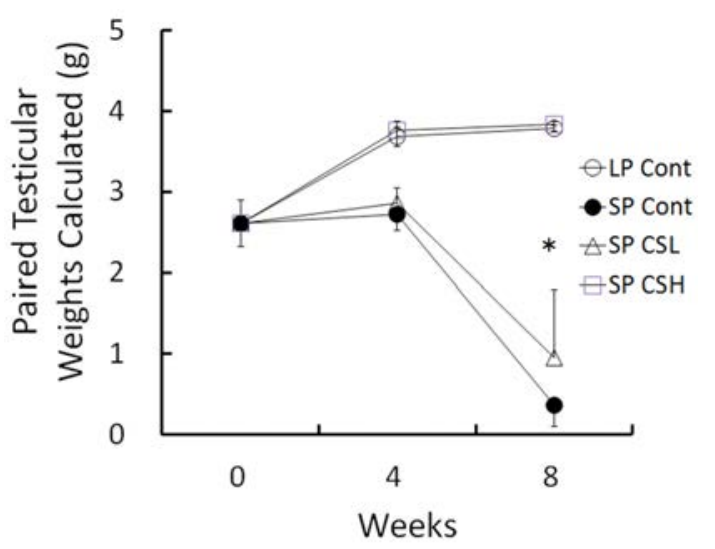

Fig. 2. Changes of testicular weights. The testicular weights of golden hamsters were gauged at 4 week intervals. Note that the testes housed in LP (LP control) held active reproductive function but the testes housed in SP (SP control) had distinctively regressed testes in 8 weeks. LP Cont; animals were maintained in LP and were treated with vehicle. SP Cont; animals were maintained in SP and were treated with vehicle. SP CSL; animals were maintained in SP and were treated with low and middle concentrations of the CS extract. SP CSH; animals were maintained in SP and were treated with high concentration of the CS extract. $n \geqq 3$. * indicates statistical significance $(\mathrm{p}<0.05)$.

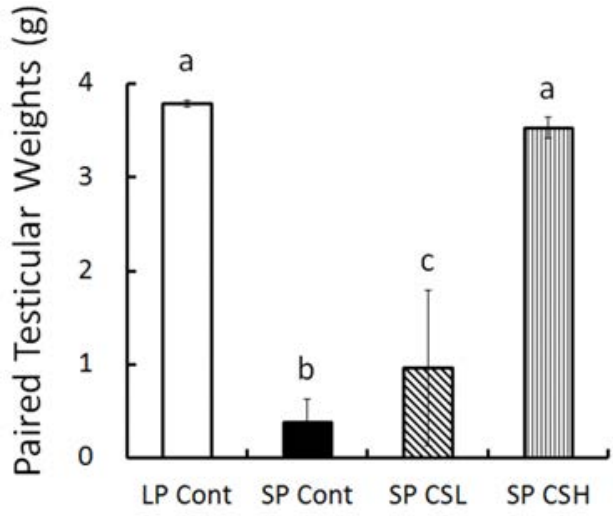

Fig. 3. Changes of real testicular weights at the end of experiment. Note that LP controls showed large testes but SP controls distinctively regressed testes in 8 weeks. LP Cont; animals were maintained in LP and were treated with vehicle. SP Cont; animals were maintained in SP and were treated with vehicle. SP CSL; animals were maintained in SP and were treated with low and middle concentrations of the CS extract. SP CSH; animals were maintained in SP and were treated with high concentration of the CS extract. $n \geqq 3$. Different letters indicate statistical significance $(\mathrm{p}<0.05)$.

control had completely involuted. The animals in SP CSL had significantly larger testes than the testes in SP control but much smaller than the testes in LP control. Astonishingly, SP CSH exhibited massive testes equivalent to the testes of animals in LP control, indicating that the CS extract blocked completely the effect of SP on the reproductive function.

Various organs were isolated and weighed at the end of experiment. Resultantly no organs were noticeably affected by the CS extract administration.

\section{DISCUSSION}

The extract of CS used in this study exhibited complete inhibition of the testicular regressing effects caused by SP in golden hamsters. To our knowledge, these results are for the first time to show the inhibitory activity of the herbal extract in blocking the regression of spermatogenesis by SP. The CS extract did not visibly affect the body weights 
and the other internal organs.

The animals housed in LP had constant large mass of testes that implied functional activity. And SP induced the complete regression of testes in golden hamsters in 8 weeks as well-known previously. The animals administered with low and middle concentration of the CS extract showed similar results and combined, which was statistically significantly different $(p<0.05)$ compared to the SP controls. Most of the animals in those groups had small testes but were uniformly a little heavier than the weights of testes of animals in SP controls. Curiously, one among the animals showed reproductively active testes with paired testicular weight of $2.8 \mathrm{~g}$, indicating total inhibition of influence of SP on reproductive endocrine system. This indicates that the animal was sensitive enough to respond to the dosage of the CS extract administered. The corresponding output was distinctly observed in the animals treated with high concentration of the CS extract. The animals had fairly large testes comparable to the testes of animals housed in LP, implying that the regressing effects of SP were completely blocked. Thus these results was thought that the CS extract prevented from the effects of SP. Yet it can not be ruled out that the complete blockade of testicular regression could be due to the somewhat insufficient period of time at which SP could act and/or due to the inferior management of animals in the facility equipped unsuitably.

The extraction methods and concentrations used in this study are grossly consistent with those reported previously. The animals received orally $0.5 \mathrm{~g}, 1.0 \mathrm{~g}, 2.5 \mathrm{~g}$ of the CS extract per $\mathrm{kg}$ of body weight in a day for 8 weeks (56 days). This method of intubation is most universal and certain. The administration time of low and middle concentrations of the CS extract was 2-3 hours before lights off and was kept at right same time of day. A careful attention was put in administering the high dose of CS extract to prevent the putative toxic effects of CS extract because the dosage had not been used in reports published up to now. The high concentration $(2.5 \mathrm{~g})$ was diluted twice and the same volumes as low and middle groups were administered in the morning and in the evening.

There is an existing report that the number of spermatozoa in the epididymis and testicular weights were significantly increased (Yang et al., 2010). Also the report presented an increase of expression of glial cell-derived neurotrophic factor (GDNF) mRNA and GDNF protein that play a pivotal role in the survival of neurons, emphasizing the improvement of reproductive function by the CS extract in males. The results of the present experiment also demonstrated an improving impact of reproductive activity. But there is a fundamental difference between the consequence of previous report and the present results. Mice are yeararound reproducing animals and golden hamsters are seasonal breeders. Although the reproductive parameters of mice in the study of Yang et al. (2010) were statistically enhanced, it is not certain that the consequences were related to the functional activity of reproduction. The fact that testicular regression by SP was blocked leads to a functional protection in the reproductive activity of golden hamsters. The discrepancy can be due to the differences of the species used or due to the exaggerated interpretation of the statistical differences. The variance can be scrutinized more precisely by measuring the reproductive hormones in blood of experimental animals. In particular, the circulatory gonadotropins, follicle stimulating hormone and luteinizing hormone secreting from the pituitary gland and testosterone secreting from the Leydig cells within the testes would reveal the cause of the differences.

The action mechanism of the CS extract could be speculated as follows no matter what animals were used. The hypothalamic control center that regulates the sexual activity in the reproductive endocrine system of mammals is composed of neuronal cells that synthesize and secrete gonadotropin releasing hormone (GnRH). These neurons affect gonadotropes in the pituitary and then the gonadotropins are released, are moved into the testes, and govern the activity of the testes.

It is very likely for the CS extract to act on the reproductive endocrine system by exerting its effect on GnRH 
neuronal cells. The CS extract swallowed by intubation into the body could be absorbed in the gastrointestinal tract and then spreaded all over the body through the circulatory system. As the steroid hormones act on the hypothalamus, some components from the CS extract could act on the hypothalamus. Also in the similar manner, they might act directly on the pituitary. It is known that SP reduces the release of GnRH via the melatonin that is synthesized and secreted from the pineal gland. Thus the CS extract possibly suppresses the action of melatonin, resulting in functional maintenance of reproductive activity in this animal. Moreover, it can be speculated that they affect directly the testes. The components of the CS extract that flowed out of the blood vessel between the seminiferous tubule of the testes operate on the Leydig cells to stimulate the synthesis of testosterone, and then the steroid immediately travels to the seminiferous tubules and instantly influences the spermatogenesis. On the other hand, it is also not irrational that the ingredients of the CS extract entered the bodily cells would be converted into another substances by the metabolic process and work on the reproductive endocrine system. But it is not yet known which elements of the CS extract operate like those mentioned above. It could be greatly helpful to further examine the hormones in blood and the expression aspects of genes to uncover those action mechanisms.

Up to date, various components have been analyzed in the CS extract. Most ingredients belong to a family of polyphenolic components such as flavonoids that lower the levels of cholesterol (Rossetti et al., 1987; Lee et al., 1999; Edwards et al., 2007; Jang et al., 2008; Egert et al., 2009; Saul et al., 2009; Tatoń et al., 2010; Yu et al., 2010). They were phlorizin, epicatechin, catechin, naringenin, naringenin4'-O-pyranogluoside, rutin, luteolin, and quercetin. The gallic acid and protocatechuic acid were detected as phenolic acids containing phenol. As phytosterols that have chemical structure similar to the cholesterol structure, $\beta$-sitosterol and daucosterol were reported (Matsuoka et al., 2008; Awad \& Fink, 2000; Moreau et al., 2002). A palmitic acid as a lipid and an ursolic acid as triterpenoid, and succinic acid were noticed (Gnoatto et al., 2008). In the light of that most of ingredients detected from the CS extract were antioxidants and reduced the values of cholesterol, it is considered that the CS extract is possibly to be involved in the reproductive function.

On the other hand, one of the efficacy of the CS extract is mounting behavior. That is, it is a breeding behavior exposed by males. If this behavior is provoked by the male sex steroid, the CS extract somehow would elevate the levels of the steroid. Many steroid variants present in the CS extract are candidate for that action but the mechanism has not been unclosed.

Taken together, the CS extract completely blocked the testicular regression influence caused by SP. But it needs further study for which ingredients have those effects and how they act within the body.

\section{REFERENCES}

Abdel-Magied EM, Abdel-Rahman HA, Harraz FM (2001) The effect of aqueous extracts of Cynomorium coccineum and Withania somnifera on testicular development in immature Wistar rats. J Ethnophamacol 75:1-4.

Abdel-Rahman HA, El-Badry AA, Mahmoud OM, Harraz FA (1999) The effect of the aqueous extract of Cynomorium cocconieum on the epididymal sperm pattern of the rat. Phytother Res 13:248-250.

Awad AB, Fink CS (2000) Phytosterols as anticancer dietary components: evidence and mechanism of action. J Nutr 130:2127-2130.

Choi D (1996) Reproductive physiology of pineal hormone melatonin. Korean J Zool 39:337-351.

Choi D, Han E-H (2010) The impacts of photoperiods on hypothalamic proteins in the reproductive activities of golden hamsters. Dev Reprod 14:185-197.

Choi D, Lee S-H (2012) Neuroendocrine system in seasonal breeder: focusing on the reproductive activity of male golden hamster. Dev Reprod 16:1-8. 
Edwards RL, Lyon T, Litwin SE, Rabovsky A, Symons JD, Jalili T (2007) Quercetin reduces blood pressure in hypertensive subjects. J Nutr 137:2405-2411.

Egert S, Bosy-Westphal A, Seiberl J, Kürbitz C, Settler U, Plachta-Danielzik S, Wagner AE, Frank J, Schrezenmeir J, Rimbach G, Wolffram S, Müller MJ (2009) Quercetin reduces systolic blood pressure and plasma oxidised lowdensity lipoprotein concentrations in overweight subjects with a high-cardiovascular disease risk phenotype: A double-blinded, placebo-controlled cross-over study. $\mathrm{Br}$ J Nutr 102:1065-1074.

Gnoatto SC, Dassonville-Klimpt A, Da Nascimento S, Galéra P, Boumediene K, Gosmann G, Sonnet P, Moslemi S (2008) Evaluation of ursolic acid isolated from Ilex paraguariensis and derivatives on aromatase inhibition. Eur J Med Chem 43:1865-1877.

Jang S, Kelley KW, Johnson RW (2008) Luteolin reduces IL-6 production in microglia by inhibiting JNK phosphorylation and activation of AP-1. Proc Natl Acad Sci USA 105:7534-7539.

Lee SH, Park YB, Bae KH, Bok SH, Kwon YK, Lee ES, Choi MS (1999) Cholesterol-lowering activity of naringenin via inhibition of 3-hydroxy-3-methylglutaryl coenzyme A reductase and acyl coenzyme A: cholesterol acyltransferase in rats. Ann Nutr Metab 43:173-180.

Matsuoka K, Nakazawa T, Nakamura A, Honda C, Endo K, Tsukada M (2008) Study of thermodynamic parameters for solubilization of plant sterol and stanol in bile salt micelles. Chem Phys Lipids 154:87-93.

Moreau RA, Whitaker BD, Hicks KB (2002) Phytosterols, phytostanols, and their conjugates in foods: structural diversity, quantitative analysis, and health-promoting uses. Prog Lipid Res 41:457-500.

Nickrent DL, Der JP, Anderson FE (2005) Discovery of the photosynthetic relatives of the "Maltese mushroom" Cynomorium. BMC Evol Biol 5:38-48.

Rossetti L, Smith D, Shulman I, Papachristou D, Defronzo A (1987) Correction of hyperglycemia with phlorizin normalizes tissue sensitivity to insulin in diabetic rats. J Clin Invest 79:1510-1515.

Saul N, Pietsch K, Menzel R, Stürzenbaum SR, Steinberg CE (2009) Catechin induced longevity in C. elegans: from key regulator genes to disposable soma. Mech Ageing Dev 130:477-486.

Tatoń J, Piatkiewicz P, Czech A (2010) Molecular physiology of cellular glucose transport - a potential area for clinical studies in diabetes mellitus. Endokrynol Pol 61:303-310.

Watson-Whitmyre M, Stetson MH (1985) A mathematical method for estimating paired testes weight from in situ testicular measurements in three species of hamster. Anat Rec 213:473-476.

Yang WM, Kim HY, Park SY, Kim H-M, Chang MS, Park SK (2010) Ethnopharmacological communication: Cynomorium songaricum induces spermatogenesis with glial cellderived neurotrophic factor (GDNF) enhancement in rat testes. J Ethnopharmacol 128:693-696.

Yu MC, Chen JH, Lai CY, Han CY, Ko WC (2010) Luteolin, a non-selective competitive inhibitor of phosphodiesterases $1-5$, displaced 3H-rolipram from high-affinity only roliprambinding sites and reversed xylazine/ketamine-induced anesthesia. Eur J Pharmacol 627:269-275.

Zhang CZ, Wang SX, Zhang Y, Chen JP, Liang XM (2005) In vitro estrogenic activities of Chinese medicinal plants traditionally used for the management of menopausal symptoms. J Ethnopharmacol 98:295-300.

(Received 14 February 2013, Received in revised form 29 February 2013, Accepted 12 March 2013) 